\title{
Micro-Raman study of stress distribution in local isolation structures and correlation with transmission electron microscopy
}

\author{
I. De Wolf, J. Vanhellemont, A. Romano-Rodriguez, ${ }^{\text {a) }}$ H. Norström, ${ }^{\text {b) }}$ and H. E. Maes \\ Interuniversity Micro-Electronics Center (IMEC), Kapeldreef 75, B-300I Leuven, Belgium
}

(Received 31 July 1991; accepted for publication 7 October 1991)

\begin{abstract}
Stress in local isolation structures is studied by micro-Raman spectroscopy. The results are correlated with predictions of an analytical model for the stress distribution and with cross-sectional transmission electron microscopy observations. The measurements are performed on structures on which the $\mathrm{Si}_{3} \mathrm{~N}_{4}$ oxidation mask is still present. The influence of the pitch of the periodic local isolation pattern, consisting of parallel lines, the thickness of the mask, and the length of the bird's beak on the stress distribution are studied. It is found that compressive stress is present in the Si substrate under the center of the oxidation mask lines, with a magnitude dependent on the width of the lines. Large tensile stress is concentrated under the bird's beak and is found to increase with decreasing length of the bird's beak and with increasing thickness of the $\mathrm{Si}_{3} \mathrm{~N}_{4}$ film.
\end{abstract}

\section{INTRODUCTION}

During the last years, micro-Raman spectroscopy has been used increasingly as a powerful technique to measure local residual stress in submicrometer isolation devices, e.g., in local oxidation of silicon (LOCOS) structures, ${ }^{1-3}$ isolation trenches, ${ }^{4}$ and V-groove structures. ${ }^{5}$ Processing of such nonplanar structures introduces large local stress, especially at discontinuities of the different film layers. Such localized stresses can induce various types of structural defects that degrade the circuit performance. The conventional LOCOS technique has been extensively employed as an isolation technique in very large-scale integration (VLSI) devices because of its simplicity. This technique is based on a selective oxidation process using silicon nitride as a mask against oxidation of the active device regions. A drawback is the diffusion of oxygen through the pad oxide during field oxidation, causing additional oxide growth under the edge of the nitride mask, giving rise to the formation of a so-called "bird's beak" (BB). This unwanted effect limits the available active surface area and, thus, the packing density. To reduce the size of the bird's beak, modified LOCOS techniques are explored with the local oxidation of polysilicon over silicon (LOPOS) technique as one of the most promising approaches. In this technique a poly-Si layer is deposited between the pad oxide and a relative thick nitride mask. The lateral diffusion of the oxide under the nitride mask is thus reduced and part of the stress induced in the silicon by the nitride mask is compensated by the poly layer. Shortening the BB leads, however, to a strong increase of the local stress in the silicon substrate. This stress is studied with micro-Raman spectroscopy and the results are compared with cross-sectional transmission electron microscopy (XTEM) and with the predictions of a simple planar stress model.

\footnotetext{
a) Present address: LCMM, Departament de Física Aplicada i Electrònica, University of Barcelona, Diagonal 645, E-08028 Barcelona, Spain.

b) Present address: Swedish Institute of Micro-Electronics, P.O. Box 1084, S-16421 Kista, Sweden.
}

\section{EXPERIMENT}

The isolation structures are fabricated on (001) silicon substrates. Experiments are performed on three different LOPOS structures and one LOCOS structure. The process parameters of the different samples are listed in Table I. In all samples a pad oxide is grown by dry oxidation. In LOPOS this is followed by deposition of poly-amorphous silicon. Next a low-pressure chemical-vapor deposition (LPCVD) $\mathrm{Si}_{3} \mathrm{~N}_{4}$ film is deposited and patterned using photolithography, resulting in long, [110]-oriented, $\mathrm{Si}_{3} \mathrm{~N}_{4}$ lines with different widths. Finally a field oxide is grown by wet oxidation at different temperatures and for different oxidation times.

Raman spectra are measured in backscattering configuration using the 488.0- or 457.9-nm line of an argon laser with output power of maximal $30 \mathrm{~mW}$. The $457.9-\mathrm{nm}$ line is used in most experiments because of its short penetration depth in $c-\mathrm{Si}$, so that only the top layer of the silicon substrate, where stress is expected to be largest, is probed during the Raman experiments. The incoming light is polarized along the length of the lines, the scattered light is, in most experiments, not analyzed. The diameter of the probed region is smaller than $1 \mu \mathrm{m}$. The sample is moved with an $X-Y$ translation stage in steps of minimum $0.1 \mu \mathrm{m}$ and at each position single-point Raman spectra are recorded. In this way the spatial variation of the Raman signal over the structures on the silicon surface is obtained. In all samples the central line of a series of five parallel lines is scanned. The spacing between these lines is equal to their width. The Raman spectra are fitted with a Lorentzian function, reducing the error on the peak position estimation to values smaller than $0.05 \mathrm{~cm}^{-1}$. Laser plasma lines are used for internal calibration.

\section{THEORY}

\section{A. Raman spectroscopy}

Crystalline silicon $(c-\mathrm{Si})$ has three active optical vibrations: two transversal $\left(\mathrm{TO}_{1}\right.$ and $\left.\mathrm{TO}_{2}\right)$ along $x=[100]$ and 
$y=[010]$ directions and one longitudinal (LO) along the $z=[001]$ direction. For strain-free $c-\mathrm{Si}$, these three vibration modes have the same frequency, $\omega_{0}$, at about 520 $\mathrm{R} \mathrm{cm}^{-1}$. They are described by the polarizability tensors ${ }^{6}$

$$
\begin{aligned}
& \mathrm{TO}_{1} \rightarrow \mathbf{R}_{1}=\left(\begin{array}{lll}
0 & 0 & 0 \\
0 & 0 & d \\
0 & d & 0
\end{array}\right), \quad \mathbf{T O}_{2} \rightarrow \mathbf{R}_{2}=\left(\begin{array}{lll}
0 & 0 & d \\
0 & 0 & 0 \\
d & 0 & 0
\end{array}\right), \\
& \mathrm{LO} \rightarrow \mathbf{R}_{3}=\left(\begin{array}{lll}
0 & d & 0 \\
d & 0 & 0 \\
0 & 0 & 0
\end{array}\right) .
\end{aligned}
$$

When incident and scattered light is polarized in the direction given by the unit vectors $\mathrm{e}_{i}$. and $\mathrm{e}_{s}$, respectively, the

$$
\left(\varphi_{i j}\right)=\left(\begin{array}{cc}
p \epsilon_{X X}+q\left(\epsilon_{Y Y}+\epsilon_{Z Z}\right) & 2 r \epsilon_{X Y} \\
2 r \epsilon_{X Y} & p \epsilon_{Y Y}+q\left(\epsilon_{Z Z}+\epsilon_{X X}\right) \\
2 r \epsilon_{Z X} & 2 r \epsilon_{Z Y}
\end{array}\right.
$$

by $\lambda_{i}=\omega_{i}^{2}-\omega_{0}^{2}, i=1,2,3$, from which follows

$$
\Delta \omega_{i}=\omega_{i}-\omega_{0} \cong \lambda_{i} / 2 \omega_{0}
$$

$\epsilon_{I J}$ are the components of the strain tensor $\epsilon$, which is related to the stress tensor $\tau$ by Hooke's law: $\epsilon=\mathbf{S} \tau$ where $\mathbf{S}$ is the compliance tensor. For $c$-Si, $\mathbf{S}$ has only three different components: $S_{11}=7.68 \times 10^{-12} \mathrm{~Pa}^{-1}, S_{12}=$ $-2.14 \times 10^{-12} \mathrm{~Pa}^{-1}$, and $S_{44}=12.7 \times 10^{-12} \mathrm{~Pa}^{-1} . p$, $q$, and $r$ are deformation potential constants ${ }^{9}$ whose values are for $c-\mathrm{Si}: p=-1.43 \omega_{0}^{2}, q=-1.89 \omega_{0}^{2}$, and $r$ $=-0.59 \omega_{0}^{2}$.

The Raman polarizability tensors also change under the influence of strain. They can be calculated by a linear combination of the zero-strain polarizability tensors, corresponding to the linear combination between old and new eigenvectors. A change of the Raman polarizability tensors often implies that the scattering efficiency changes and that besides $\mathrm{LO}$ also $\mathrm{TO}_{1}$ and/or $\mathrm{TO}_{2}$ may contribute to the signal.

TABLE I. Processing parameters of the samples. Nominal thicknesses are indicated in $\mathrm{nm}$.

\begin{tabular}{lccccc}
\hline \hline Sample & $\begin{array}{c}\mathrm{Si}_{3} \mathrm{~N}_{4} \\
\text { thickness }\end{array}$ & $\begin{array}{c}\text { Pad oxide } \\
\text { thickness }\end{array}$ & $\begin{array}{c}\text { Field oxide } \\
\text { thickness }\end{array}$ & $\begin{array}{c}\text { Poly-Si } \\
\text { thickness }\end{array}$ & $T\left({ }^{\circ} \mathrm{C}\right)$ \\
\hline LOPOS 1 & 150 & 10 & 600 & 50 & 1000 \\
LOCOS 1 & 150 & 10 & 600 & $\ldots$ & 950 \\
LOPOS 2 & 156 & 18 & 700 & 50 & 950 \\
LOPOS 3 & 250 & 18 & 700 & 50 & 950 \\
\hline \hline
\end{tabular}

scattering efficiency (scattered power divided by incident power) of the Raman signal is given by

$$
S=A \sum_{j}\left|\mathrm{e}_{i} \cdot \mathbf{R}_{j} \cdot \mathrm{e}_{s}\right|^{2},
$$

where $A$ is a constant. In the case of backscattering from a (001) surface, the $z$ component of $\mathbf{e}_{i}$ and $\mathbf{e}_{s}$ is zero, so that only $\mathbf{R}_{\mathbf{3}}$ contributes to the Raman signal. Only the LO phonon can be observed.

Strain lowers the symmetry of the crystal so that the degeneracy of the three optical phonons is partly lifted and they shift to frequencies different from $\omega_{0}$. The phonon frequencies and polarizability tensors of strained silicon can be calculated using perturbation theory. ${ }^{7,8}$ The frequencies $\omega_{i}$ of the optical phonons in the presence of strain are related to the eigenvalues $\lambda_{i}$ of the following secular matrix $\left(\varphi_{i j}\right)$

$\left.\begin{array}{c}2 r \epsilon_{X Z} \\ 2 r \epsilon_{Y Z} \\ p \epsilon_{Z Z}+q\left(\epsilon_{X X}+\epsilon_{Y Y}\right)\end{array}\right)$

\section{B. Stress model}

The calculation of the eigenvalues of the secular matrix results in relations between the shift of each optical phonon and the strain in the probed sample, as described by Eqs. (3) and (4). In the most general case, when none of the strain tensor components is zero, this will yield for each vibration mode a complicated relation between the shift of the mode and nine different strain tensor components. In such a case it is difficult to obtain information on the strain present in the sample. For this reason it is necessary to make some assumptions about strain and strain distribution in the investigated sample. These assumptions can then be evaluated using the results of the Raman experiments.

It is not straightforward to derive an analytical model that describes the strain in the silicon underneath a LOCOS or LOPOS structure. The most simple assumption is a model where the structure is replaced by a patterned layer-substrate structure. The mechanical stress caused by a thin film on a substrate was studied by several authors. ${ }^{10-13}$ It was found that in general the induced stress is small in the substrate, due to its much larger thickness compared to that of the film. However, in the neighborhood of discontinuities in the film, large localized stresses are generated in the substrate. This film-edge-induced stress can be described by an edge force $f$, with origin at the film edge, and acting parallel to the surface of the film, as is shown in Fig. 1(a). For a line of width $w$, two opposed forces with equal magnitude determine the stress induced in the substrate. Assuming planar stress, the stress components in the substrate are then given by ${ }^{12,13}$ 
(a)

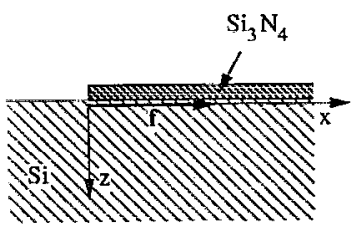

(b)

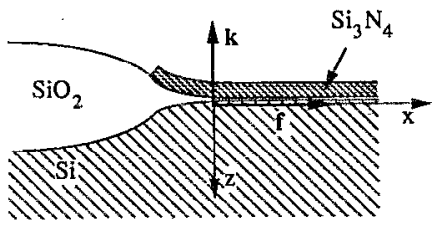

FIG. 1. Cross-sectional view of (a) a $\mathrm{Si}_{3} \mathrm{~N}_{4}$ line and (b) a LOCOS structure with forces acting on the substrate.

$$
\begin{aligned}
& \sigma_{x x}=-\frac{2 f}{\pi}\left(\frac{x^{3}}{\left(x^{2}+z^{2}\right)^{2}}-\frac{(x-w)^{3}}{\left[(x-w)^{2}+z^{2}\right]^{2}}\right), \\
& \sigma_{z z}=-\frac{2 f}{\pi} z^{2}\left(\frac{x}{\left(x^{2}+z^{2}\right)^{2}}-\frac{(x-w)}{\left[(x-w)^{2}+z^{2}\right]^{2}}\right), \\
& \tau_{x z}=-\frac{2 f}{\pi} z\left(\frac{x^{2}}{\left(x^{2}+z^{2}\right)^{2}}-\frac{(x-w)^{2}}{\left[(x-w)^{2}+z^{2}\right]^{2}}\right) .
\end{aligned}
$$

In the case of LOCOS or LOPOS structures, the $\mathrm{Si}_{3} \mathrm{~N}_{4}$ film is pushed upwards at the edges by the field oxide that grows laterally underneath the film during oxidation. The film model does not account for this bending up. It results in large tensile stresses on the silicon substrate in the neighborhood of the BBs end, because the BB acts as if it wedges in between the film and the substrate, trying to separate both. Vanhellemont and co-workers ${ }^{13}$ introduced a model in which this effect was accounted for by introducing a vertical acting force, $\mathbf{k}=\alpha \mathbf{f}$, with origin at the BBs edge, in addition to the horizontal edge force $f$. This is shown in Fig. 1(b). This model was successfully used to describe film-edge-induced dislocation generation in LOCOS structures. For a single isolated $\mathrm{Si}_{3} \mathrm{~N}_{4}$ line the stress components are given by

$\sigma_{x x}=-\frac{2 f}{\pi}\left(\frac{x^{2}(x+\alpha z)}{\left(x^{2}+z^{2}\right)^{2}}-\frac{(x-w)^{2}(x-w-\alpha z)}{\left[(x-w)^{2}+z^{2}\right]^{2}}\right)$,

$\sigma_{z z}=-\frac{2 f}{\pi} z^{2}\left(\frac{(x+\alpha z)}{\left(x^{2}+z^{2}\right)^{2}}-\frac{(x-w-\alpha z)}{\left[(x-w)^{2}+z^{2}\right]^{2}}\right)$,

$\tau_{x z}=-\frac{2 f}{\pi} z\left(\frac{x(x+\alpha z)}{\left(x^{2}+z^{2}\right)^{2}}-\frac{(x-w)(x-w-\alpha z)}{\left[(x-w)^{2}+z^{2}\right]^{2}}\right)$,

where $w$ indicates the distance between the two bird's beak tips. Figure 2 shows the dependence of $\sigma_{x x} / f, \sigma_{z z} / f$, and $\tau_{x z} / f$ on the position near or under a line for $z=0.2 \mu \mathrm{m}$, $\alpha=-0.3$, and $w=5 \mu \mathrm{m}$. As can be seen, both $\sigma_{x x}$ and $\sigma_{z z}$ are compressive under the lines, in between the BBs ends. Equations (8) $-(10)$ are only valid for isolated lines. For a periodic structure consisting of $n$ parallel $\mathrm{Si}_{3} \mathrm{~N}_{4}$ lines with spacing $s$ and width $w$, the parameter $x$ in these equations must be replaced by $[x-n(w+s)]$ and a summation over $n$ must be made. ${ }^{13}$

In all samples studied, the $\mathrm{Si}_{3} \mathrm{~N}_{4}$ lines are oriented along a [110] direction. This means that Eqs. (8)-(10) are valid in the axes system where the $x$ axis is oriented along the crystallographic direction [110], the $y$ axis along [110],
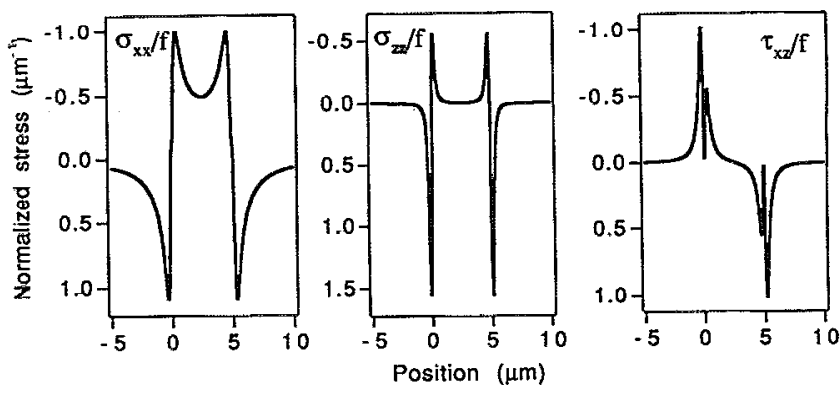

FIG. 2. The stress components $\sigma_{x x} / \int, \sigma_{z z} / \int$, and $\tau_{x z} / f$ in the silicon substrate under a LOCOS structure. The components were calculated using Eqs. (7)-(9) and parameters: $z=0.2 \mu \mathrm{m}, \alpha=-0.3$, and $w=5$ $\mu \mathrm{m}$.

and the $z$ axis along [00피 (sample system). The secular matrix is given in an axes system (reference system, indices are caps) where the $X$ axis is directed along the [100] crystallographic direction, the $Y$ axis along [010], and the $Z$ axis along [001]. The stress components in both systems are related by

$$
\left\{\begin{array} { l } 
{ \sigma _ { X X } = \frac { \sigma _ { x x } } { 2 } + \frac { \sigma _ { y y } } { 2 } - \tau _ { x y } = \frac { \sigma _ { x x } } { 2 } } \\
{ \sigma _ { Y Y } = \frac { \sigma _ { x x } } { 2 } + \frac { \sigma _ { y y } } { 2 } + \tau _ { x y } = \frac { \sigma _ { x x } } { 2 } } \\
{ \sigma _ { z Z } = \sigma _ { z z } }
\end{array} \left\{\begin{array}{l}
\tau_{X Y}=\frac{\sigma_{x x}}{2}-\frac{\sigma_{y y}}{2}=\frac{\sigma_{x x}}{2} \\
\tau_{Y Z}=\frac{\tau_{y z}}{\sqrt{2}}+\frac{\tau_{x z}}{\sqrt{2}}=\frac{\tau_{x z}}{\sqrt{2}} \\
\tau_{X Z}=-\frac{\tau_{y z}}{\sqrt{2}}+\frac{\tau_{x z}}{\sqrt{2}}=\frac{\tau_{x z}}{\sqrt{2}}
\end{array},\right.\right.
$$

where plane stress (only $\sigma_{x x}, \sigma_{z z}$, and $\tau_{x z}$ differ from zero) is assumed. So none of the stress components is zero in the reference system, $\sigma_{X X}=\sigma_{Y Y}=\tau_{X Y}$ and $\tau_{Y Z}=\tau_{X Z}$. From this it follows that the strain tensor components that have to be used in the secular matrix become (Hooke's law)

$$
\begin{aligned}
& \epsilon_{X X}=\epsilon_{Y Y}=\left(S_{11}+S_{12}\right) \sigma_{X X}+S_{12} \sigma_{Z Z}, \\
& \epsilon_{Z Z}=2 S_{12} \sigma_{X X}+S_{11} \sigma_{Z Z}, \\
& \epsilon_{X Y}=\left(S_{44} / 2\right) \sigma_{X X}, \\
& \epsilon_{Y Z}=\epsilon_{X Z}=\left(S_{44} / 2\right) \tau_{X Z} .
\end{aligned}
$$

The reference system stress components in Eq. (12) can then be replaced by the sample system stress components using Eqs. (11). In this way, the elements of the secular matrix [Eq. (3)], $\varphi_{i j}$, can be expressed in the sample system. None of the nine matrix elements is zero. Solving this matrix gives three different eigenvalues and corresponding eigenvectors. From the eigenvalues $\lambda_{1}, \lambda_{2}$, and $\lambda_{3}$ the following dependence of the frequency shift $\Delta \omega_{1}, \Delta \omega_{2}$, and $\Delta \omega_{3}$ of the three Raman peaks (peak 1, peak 2, and peak 3, respectively) on $\sigma_{x x}, \sigma_{z z}$ and $\tau_{x z}$ [Eq. (4)] was obtained:

$$
\begin{aligned}
& \Delta \omega_{1}=\left(\varphi_{11}-\varphi_{12}\right) /\left(2 \omega_{0}\right), \\
& \Delta \omega_{2}=\left(\varphi_{11}+\varphi_{12}+\varphi_{33}+\sqrt{D}\right) /\left(4 \omega_{0}\right), \\
& \Delta \omega_{3}=\left(\varphi_{11}+\varphi_{12}+\varphi_{33}-\sqrt{D}\right) /\left(4 \omega_{0}\right),
\end{aligned}
$$


where

$$
D=\left(\varphi_{11}+\varphi_{12}-\varphi_{33}\right)^{2}+8\left(\varphi_{13}\right)^{2}
$$

and corresponding eigenvectors

$$
\nabla_{1}=\left(\begin{array}{l}
1 \\
-1 \\
0
\end{array}\right), \quad \nabla_{2}=\left(\begin{array}{l}
1 \\
1 \\
A
\end{array}\right), \quad \nabla_{3}=\left(\begin{array}{l}
1 \\
1 \\
B
\end{array}\right),
$$

where •

$$
\begin{aligned}
& A=\left(-\varphi_{11}-\varphi_{12}+\varphi_{33}+\sqrt{D}\right) /\left(2 \varphi_{13}\right), \\
& B=\left(-\varphi_{11}-\varphi_{12}+\varphi_{33}-\sqrt{D}\right) /\left(2 \varphi_{13}\right) .
\end{aligned}
$$

From these eigenvectors the polarizability tensors in the presence of these stresses were calculated:

$$
\begin{aligned}
& \mathbf{R}_{1}=\left(\begin{array}{lll}
0 & 0 & -d \\
0 & 0 & d \\
-d & d & 0
\end{array}\right), \\
& \mathbf{R}_{2}=\frac{1}{\sqrt{2+A^{2}}}\left(\begin{array}{ccc}
0 & A d & d \\
A d & 0 & d \\
d & d & 0
\end{array}\right), \\
& \mathbf{R}_{3}=\frac{1}{\sqrt{2+B^{2}}}\left(\begin{array}{ccc}
0 & B d & d \\
B d & 0 & d \\
d & d & 0
\end{array}\right) .
\end{aligned}
$$

This result implies that, depending on the value of $A$ and $B$, both the Raman signal corresponding with peak 2 and the one corresponding with peak 3 can be observed, while peak 1 is not visible [Eq. (2) assuming back scattering]. Figure 3 shows the expected frequency shift [Fig. 3(a)] and intensity [Fig. 3(b)] of both Raman peaks at various positions across the LOCOS structures. The same values for $z, \alpha$ and $w$ were used as in Fig. $2, f=700 \mathrm{~N} / \mathrm{m}$. From these simulations it follows that the Raman signal obtained at the center of the lines is dominated by peak 3 , while the signal outside the lines is mainly determined by peak 2 .

\section{RESULTS AND DISCUSSION}

\section{A. 3- $\mu \mathrm{m}$ LOPOS line}

In between the measurements on LOPOS or LOCOS structures discussed in this paper, the Raman signal of a stress-free $c$-Si reference sample was also recorded. From nine experiments a value of $\omega_{0}=520.0 \mathrm{R} \mathrm{cm}^{-1}$ (s.d. $=0.19$ ) was obtained. This value is used in the following experiments as reference value for unstressed $c$-Si.

Figure 4(a) shows the frequency of the $c$-Si Raman signal that was measured during a scan across a $3-\mu \mathrm{m}$ $\mathrm{Si}_{3} \mathrm{~N}_{4}$ mask line (LOPOS 1) as a function of the position across the line. The Raman signal was obtained by focusing the laser light with wavelength $457.9 \mathrm{~nm}$ on the Si substrate. $\mathrm{SiO}_{2}$ and $\mathrm{Si}_{3} \mathrm{~N}_{4}$ are transparent for this wavelength and neither of them gives a measurable contribution to the Raman signal, so that only the signal from the underlying substrate is obtained. The contribution of the poly-Si to the Raman signal was controlled by measuring the polariza-
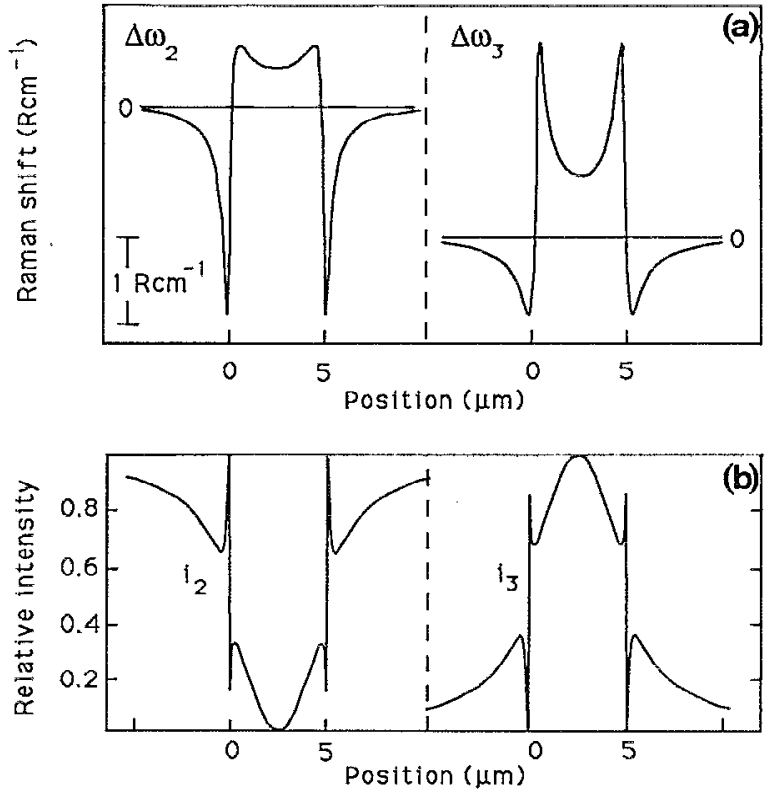

FIG. 3. (a) The theoretically expected frequency shift $\Delta \omega$ and (b) intensity $i$ of Raman peaks 2 and 3 at various positions across a LOCOS structure. The frequency shift of the peaks is calculated using Eqs. (13)(15). $\Delta \omega_{1}$ is not shown because this peak is not visible $\left(i_{1}=0\right)$. The intensity of the peaks is calculated using Eq. (2) where the polarizability tensors are given by Eq. (17) and with the assumption of backscattering. The same values for $z, \alpha$, and $w$ were used as in Fig. $2 ; f=700 \mathrm{~N} / \mathrm{m}$.

tion direction sensitivity of the scattered light. It was found to be negligible. So the main Raman signal arises from an integrated area of about $0.3 \mu \mathrm{m}$ (the penetration depth of 457.9-nm light in $c$-Si) in the substrate. A cross-sectional TEM micrograph of the corresponding LOPOS geometry

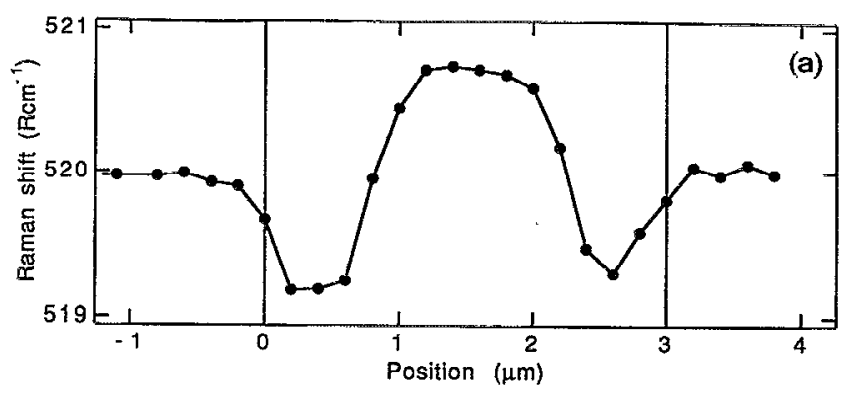

(b)

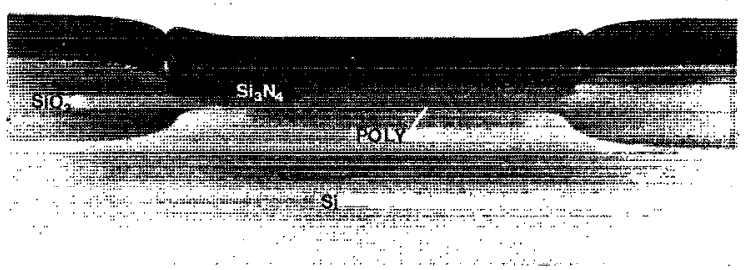

FIG. 4. (a) Frequency of the Raman signal that was measured during a scan across a $3-\mu \mathrm{m} \mathrm{Si}_{3} \mathrm{~N}_{4}$ line (LOPOS 1). Wavelength of the laser light: $457.9 \mathrm{~nm}$. The vertical lines denote the borders of the $\mathrm{Si}_{3} \mathrm{~N}_{4}$ mask. (b) A cross-sectional TEM micrograph showing the geometry of the same sample. 
is shown in Fig. 4(b). A polysilicon layer was depositcd on the sample to improve the contrast of the picture. The horizontal scale of Figs. 4(a) and $4(\mathrm{~b})$ is equal so that a direct correlation can be made between the frequency of the Raman signal and the geometrical area in the LOPOS from which the signal arises.

The frequency of the Raman signal arising from the $c$-Si substrate underneath the field oxide $\left(\omega_{o x}\right)$ far from the line edges (position $<-0.6 \mu \mathrm{m}$ and $>3.4 \mu \mathrm{m}$ ) was found to be very near to $520 \mathrm{R} \mathrm{cm}^{-1}$ indicating that the substrate is stress free at this position. Approaching the $\mathrm{Si}_{3} \mathrm{~N}_{4}$ line borders from the field oxide side leads to a frequency shift towards lower values, reaching a minimum in the vicinity of the BB edge. This downward shift from $\omega_{0}$ is large and indicative for high tensile stress in the substrate at the BBs edge. It is important to notice that this minimum is not located at the edge of the $\mathrm{Si}_{3} \mathrm{~N}_{4}$ line, but at the BBs end, underneath the $\mathrm{Si}_{3} \mathrm{~N}_{4}$ line. The Raman frequency increases again towards the center of the line and reachcs a maximum of about $520.7 \mathrm{R} \mathrm{cm}^{-1}$ at the center. This frequency is higher than the frequency of stress-free $c$-Si and indicates the presence of compressive stress in the top layer of the silicon substrate at this position. The experimentally obtained Raman shift shows roughly the same behavior as the shift predicted by the model [Fig. 3(b)]. According to the theory, the relative intensity of peak 2 is maximal outside the $\mathrm{Si}_{3} \mathrm{~N}_{4}$ lines and the intensity of peak 3 becomes zero at this position [Fig. 3(a)]. This indicates that only peak 2 is visible in the Raman spectrum and that the relation between the Raman shift and stress should be calculated according to $\mathrm{Eq}$. (14). At the center of the $\mathrm{Si}_{3} \mathrm{~N}_{4}$ lines, peak 3 has maximal and peak 2 minimal intensity so that the Raman signal is dominated by peak 3 and the relation between Raman shift and stress is given by Eq. (15). This makes an accurate fit of the proposed model to the Raman data impossible. According to the model, both peaks have nearly equal intensity and should be visible at the edge of the BB. When both peaks are present, they can only be resolved when their relative shift, which is equal to $2 C$ according to Eqs. (14)-(15), is higher than their full width at half-maximum (FWHM). Otherwise, if $2 C$ $<$ FWHM, the superposition of these two peaks will result in a broadened peak. We never observed two different peaks in this area. However, a significant increase of the FWHM of the $c$-Si Raman signal in the vicinity of the BBs edge was indeed measured. This may suggest the presence of two unresolved Raman peaks. However this increase of the FWHM may also be caused by spatial inhomogeneity of the stress within the laser-beam diameter and within the probed depth. The steep variation of the Raman frequency measured at the BBs edge indeed confirms the presence of a large stress gradient in this area.

\section{B. Influence of linewidth}

To study the influence of the $\mathrm{Si}_{3} \mathrm{~N}_{4}$ linewidth on the Raman frequency, we repeated a similar measurement as shown in Fig. 4 for lines with widths varying from 15 to 2 $\mu \mathrm{m}$. Figure 5 shows the shift of the Raman frequency from $\omega_{\mathrm{ox}}$ for different positions along the width of the lines of
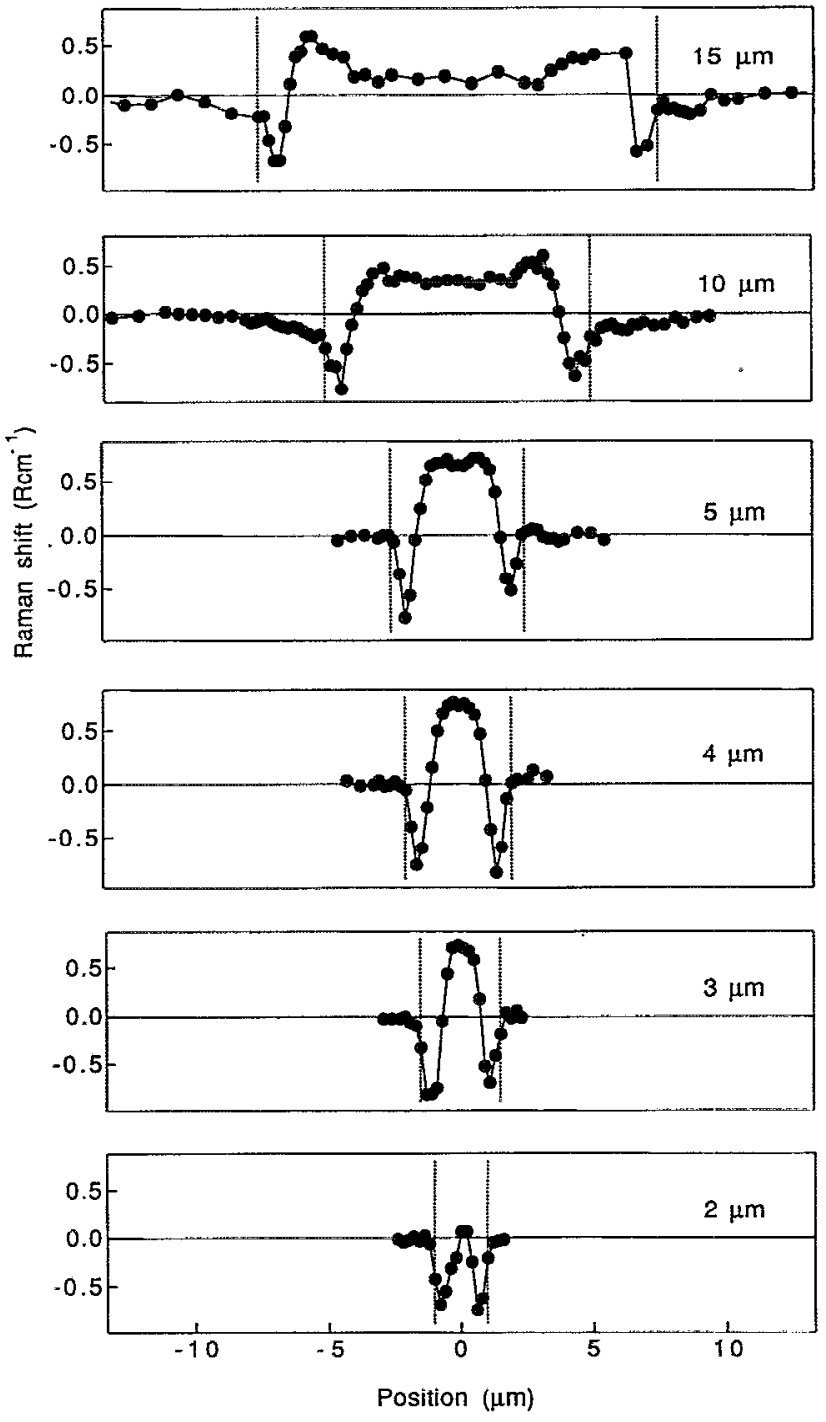

FIG. 5. Difference between the Raman frequency measured along the width of lines of the LOPOS 1 sample and $\omega_{\mathrm{ox}}$ for lines with different $\mathrm{Si}_{3} \mathrm{~N}_{4}$ linewidths $(2-15 \mu \mathrm{m})$. The vertical lines denote the borders of the $\mathrm{Si}_{3} \mathrm{~N}_{4}$ mask.

LOPOS 1. For broad lines (5-15 $\mu \mathrm{m})$, the maximum shift is not reached at the center of the lines, but closer to the borders, indicating a relaxation of the compressive stress toward the center of the line. This relaxation is predicted by the model, as is shown in Fig. 3(a). To obtain information about the magnitude of these local stresses, we focused on the Raman shift in the center of the lines $\left(\Delta \omega_{\text {center }}\right)$ and the most negative shift $\left(\Delta \omega_{\min }\right)$ in the vicinity of the BBs edge. Both were calculated relative to the Raman shift measured in between the lines $\left(\omega_{\mathrm{ox}}\right)$. Figure 6 shows the mean values of $\Delta \omega_{\min }$ and $\Delta \omega_{\text {center }}$ obtained for different linewidths.

$\Delta \omega_{\text {center }}$ increases with decreasing linewidth, up to 4 $\mu \mathrm{m}$, but decreases for smaller lines. According to the theory, $\tau_{x z}$ is zero and $\sigma_{z z}$ is negligible compared with $\sigma_{x x}$ at the center of the line for broad lines $(>4 \mu \mathrm{m}$ ) (see Fig. 2), so that uniaxial stress (only $\sigma_{x x}$ differs from zero) can be 


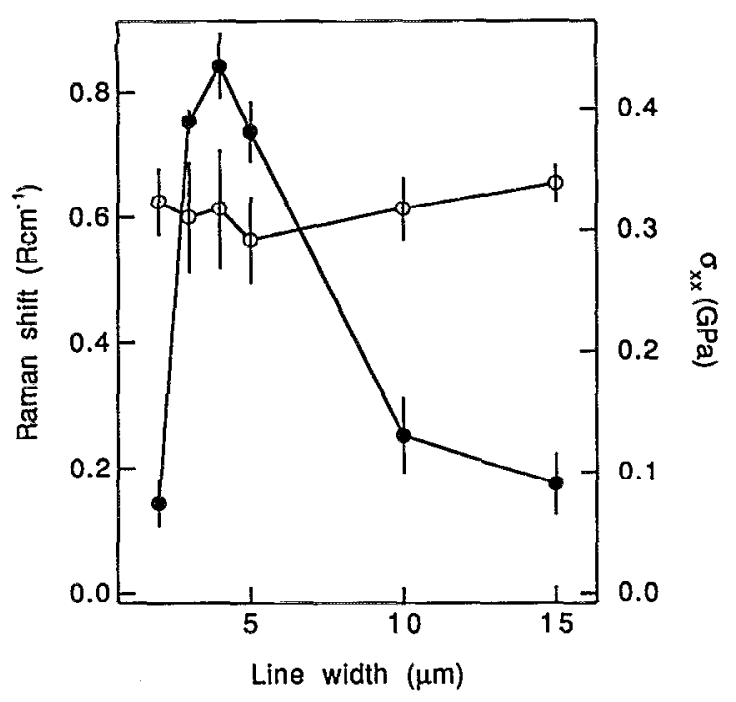

FIG. 6. The mean value of the Raman shift in the center of the lines $\left(\Delta \omega_{\text {center }}:-0_{-}\right)$and the most negative shift $\left(\Delta \omega_{\min }:-O_{-}\right)$in the vicinity of the BBs edge as a function of linewidth. Both were calculated relative to $\omega_{0 \mathrm{x}}$. The right $Y$ axis denotes the stress calculated assuming uniaxial stress in the center of the lines. These values are only valid for broad lines (15-4 $\mu \mathrm{m})$.

assumed. Furthermore, at the line center the intensity of peak 2 is negligible compared to the intensity of peak 3 [Fig. 3(b)] so that peak 3 dominates the spectrum. In this case Eq. (14) can be used to calculate the relation between Raman shift and stress. Assuming $\tau_{x z}$ and $\sigma_{z z}$ equal to zero this equation simplifies to $\left(\varphi_{13}=0\right)$ :

$$
\begin{aligned}
\Delta \omega_{3} & =\varphi_{33} / 2 \omega_{0} \\
& =\left(1 / 2 \omega_{0}\right)\left[p S_{12}+q\left(S_{11}+S_{12}\right)\right] \sigma_{x x} \\
& =-1.93 \times 10^{-9} \sigma_{x x} .
\end{aligned}
$$

The same equation is obtained when the eigenvalues of the secular equation are solved under the assumption of uniaxial stress along $[110] .{ }^{14}$ Using this equation, $\sigma_{x x}$ was calculated from $\Delta \omega_{\text {center. }}$. The values varied from about -0.09 $\mathrm{GPa}$ in the center of $15-\mu \mathrm{m}$ lines to $-0.4 \mathrm{GPa}$ in the center of $4-\mu \mathrm{m}$ lines. For broad lines the term $\alpha z$ in Eq. (8) is much smaller than $w$ and can be neglected. Using the above-obtained values for $\sigma_{x x}$, Eq. (8) can be solved for the factor $f$, yiclding $f=500 \mathrm{~N} / \mathrm{m}$. This valuc corresponds well with $f=800 \mathrm{~N} / \mathrm{m}$ estimated from computer simulations of the pile-up configuration of $60^{\circ}$ dislocations in similar structures. ${ }^{15}$

For smaller lines $\tau_{x z}$ and $\sigma_{z z}$ cannot be neglected any more and Eq. (18) is no longer valid. We attribute the decrease of the Raman shift in the center of these lines to an increasing influence of the tensile stress at the BBs edge caused by the bending up of the $\mathrm{Si}_{3} \mathrm{~N}_{4}$ film.

Figure 7 shows the theoretical dependence of $\Delta \omega_{\min }$ on some of the parameters used in the model: $f$ (at $\alpha=-0.3$ and -0.6 ), $\alpha$ (at $f=700 \mathrm{~N} / \mathrm{m}$ ), and linewidth $w$ (at $f=700 \mathrm{~N} / \mathrm{m}$ and $\alpha=-0.3$ ). The figure shows that the model predicts a strong dependence of $\Delta \omega_{\min }$ on $f$ and $\alpha$, but almost no dependence on the linewidth $w$. We indeed
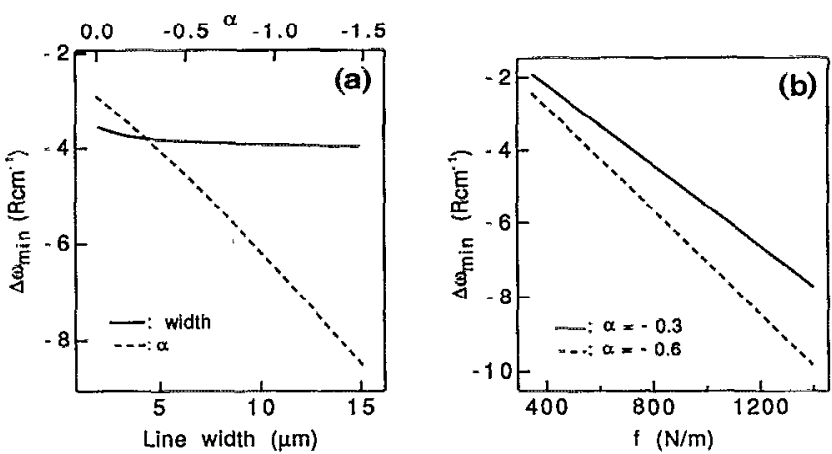

FIG. 7. Theoretical dependence of $\Delta \omega_{\min }$ on some of the parameters used in the model: (a) on line width ( $w$, full line) (at $f=700 \mathrm{~N} / \mathrm{m}$ and $\alpha=-0.3$ ) and on $\alpha$ (dashed line) (at $f=700 \mathrm{~N} / \mathrm{m}$ and $w=5 \mu \mathrm{m}$ ); (b) on force $f$ [at $\alpha=-0.3$ (full line) and -0.6 (dashed line)]

found that $\Delta \omega_{\min }$ did not change with $w$ (Fig. 6). This also indicates that $f$ as well as $\alpha$ are independent of linewidth.

The shift measured at the center of the field oxide region in between the lines $\left(\omega_{\mathrm{ox}}\right)$ was found to be slightly dependent on line spacing and much smaller (maximal 0.1 $\mathrm{R} \mathrm{cm}{ }^{-1}$ ) than the shift observed underneath the nitride lines. ${ }^{16}$ For this reason $\omega_{\mathrm{ox}}$ could be used as reference in the Figs. 5 and 6 . In all measured samples the shift was zero or slightly negative with regard to $\omega_{0}$, indicating small tensile stresses $(0.017 \mathrm{GPa}$ between $15-\mu \mathrm{m}$ lines, $0.064 \mathrm{GPa}$ between $4-\mu \mathrm{m}$ lines). Tensile stress in the substrate underneath the field oxide is expected from the difference in thermal-expansion coefficients of $c$-Si and $\mathrm{SiO}_{2}{ }^{17}$ Detailed micro-Raman experiments on these stresses were reported by Brunner et al. ${ }^{3}$

Kobayashi et al. ${ }^{1}$ reported micro-Raman experiments on LOCOS structures after removal of the $\mathrm{Si}_{3} \mathrm{~N}_{4}$ film. Their results show that the shift of the Raman frequency to values lower than the stress-free value was not observed at the BBs edge, at a position in the active area, but outside the active area at the field oxide edges. We performed Raman experiments on a LOCOS sample, ${ }^{16}$ before and after removal of the $\mathrm{Si}_{3} \mathrm{~N}_{4}$ mask, and found a similar shift: With the mask, the minimum Raman frequency was reached at the edge of the BB, without mask, $\Delta \omega_{\min }$ was smaller and the minimum was reached outside the former borders of the $\mathrm{Si}_{3} \mathrm{~N}_{4}$ line, probably in the area of the bending in the $\mathrm{SiO}_{2} / \mathrm{Si}$ interface [which can be observed in Figs. 9(b) and 9 (c)]. Without mask, the stress is mainly determined by the difference between the thermal-expansion coefficients of $\mathrm{SiO}_{2}$ and $c-\mathrm{Si}$ and by the volume expansion of $\mathrm{SiO}_{2}$ during field oxidation. Kobayashi et al. ${ }^{1}$ observed an increase of $\Delta \omega_{\text {center }}$ with decreasing linewidth down to widths as small as $1.2 \mu \mathrm{m}$, which is in contrast with the results we obtained when the $\mathrm{Si}_{3} \mathrm{~N}_{4}$ film is present. These differences can be explained by the influence of the bending up of the $\mathrm{Si}_{3} \mathrm{~N}_{4}$ film. This causes large tensile stress in the silicon at the edge of the BB. This stress disappears when the film is removed and the influence of the field oxide on stress becomes dominant. For small linewidths, the influence of this bending of the film may extend to the center of the lines, 


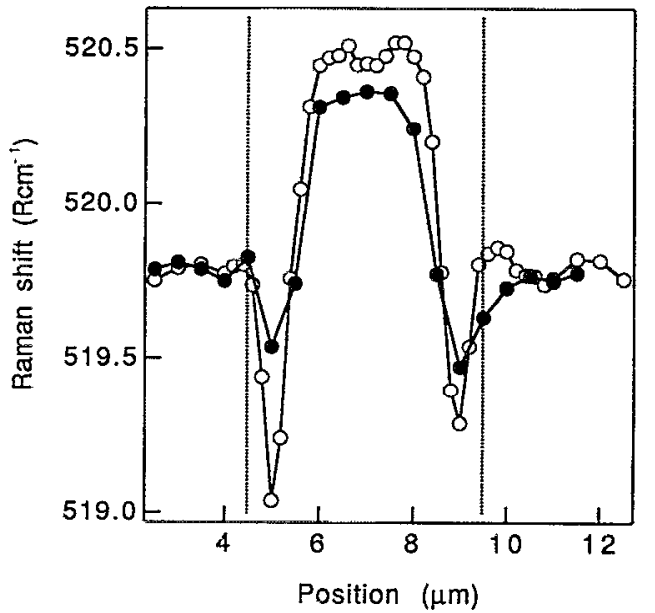

FIG. 8. Influence of laser light wavelength used for excitation of the Raman signal on the Raman shift observed during a scan across a $5-\mu \mathrm{m}$ LOPOS 2 line. - $-: 488.0 \mathrm{~nm} ;-0-: 457.9 \mathrm{~nm}$. The vertical lines indicate the borders of the $\mathrm{Si}_{3} \mathrm{~N}_{4}$ mask.

causing the decrease of $\Delta \omega_{\text {center }}$ for small lines. The present simple stress model is not accurate enough to predict this effect, mainly because we only use one vertical force component $\mathbf{k}$. It is likely that a continuous vertical force distribution, with decreasing magnitude toward the center of the lines, is more realistic. However, such a model would become too complicated to predict the Raman shift.

\section{Influence of light penetration depth}

The penetration depth of light increases with increasing wavelength. The Raman signal obtained with $457.9 \mathrm{~nm}$ arises from an integrated area with depth of about $0.3 \mu \mathrm{m}$. Light with a wavelength of $488 \mathrm{~nm}$ will give information about an integrated area of about $0.6 \mu \mathrm{m}$. According to the model, the stress in the silicon substrate decreases with depth $[z$ in Eqs. (8) $-(10)]$ and this stress gradient is predicted to be larger at the BBs edge than at the center of the lines. Figure 8 shows the dependence of the Raman frequency on the position across a $5-\mu \mathrm{m}$ LOPOS line (LOPOS 1), measured with 457.9- and 488.0-nm excitation light wavelengths. At $457.9 \mathrm{~nm} \Delta \omega_{\text {center }}$ and $\Delta \omega_{\min }$ are clearly larger than the shifts measured with $488 \mathrm{~nm}$, indicating higher stress near the surface of the substrate. Also, as predicted by the model, the influence of the excitation light wavelength on Raman shift is larger at the BB than in the center of the line. These results show that micro-Raman spectroscopy also can provide some information on the depth distribution of stress.

\section{Influence of $\mathrm{Si}_{3} \mathbf{N}_{\mathbf{4}}$ line thickness}

As is shown in Fig. 7, the stress model predicts that $\Delta \omega_{\min }$ decreases when $\alpha$ becomes more ncgative and thus when the vertical force $\mathbf{k}=\alpha \mathbf{f}$ increases. This force accounts for the tensile stress induced in the $c$-Si substrate by the bended $\mathrm{Si}_{3} \mathrm{~N}_{4}$ film. Thicker $\mathrm{Si}_{3} \mathrm{~N}_{4}$ films are more resistant to this bending and therefore induce a higher tensile
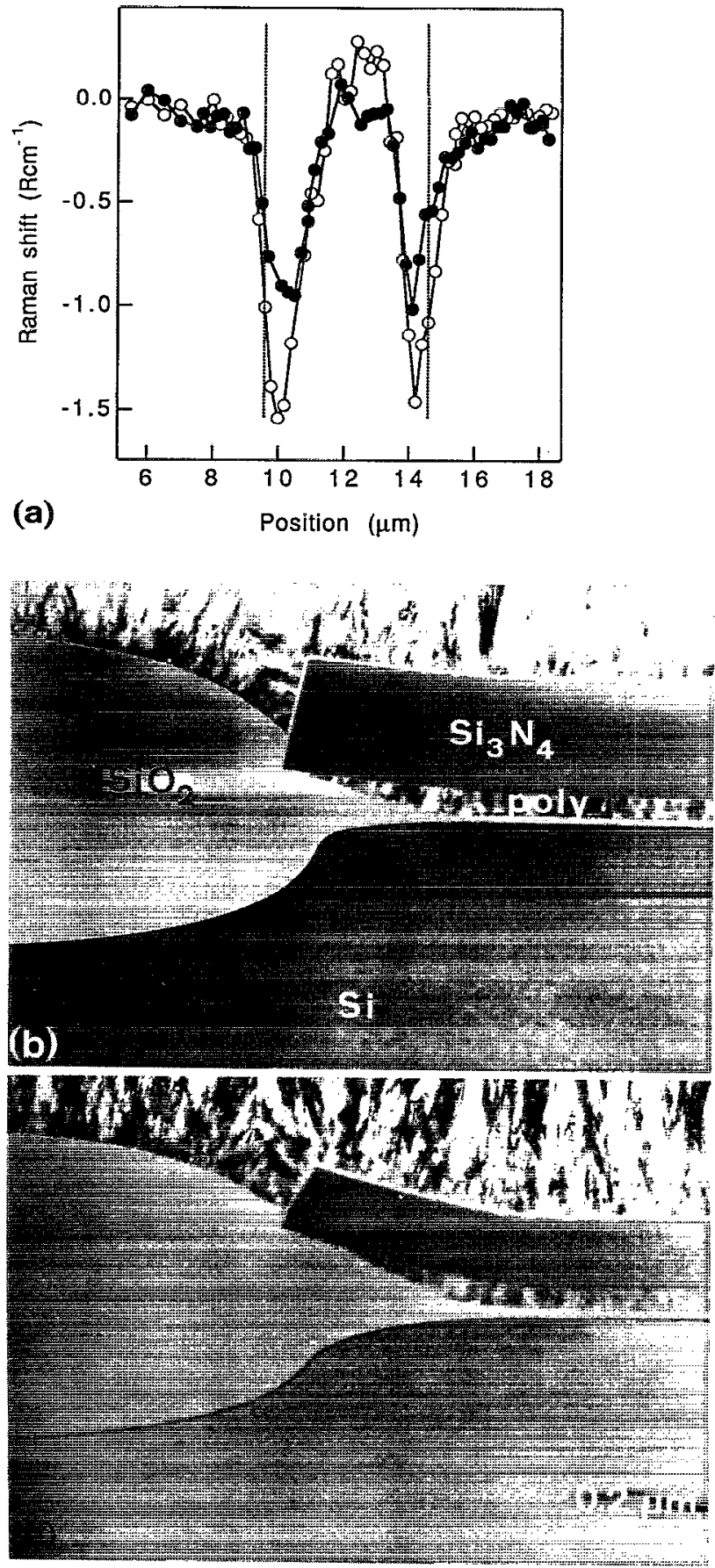

FIG. 9. (a) Raman frequency shift obtained during a scan across a $5-\mu \mathrm{m}$ line of the LOPOS 2 sample (- -; thickness $\mathrm{Si}_{3} \mathrm{~N}_{4}$ film $=156 \mathrm{~nm}$ ) and the LOPOS 3 sample ( $-\mathrm{O}_{-}$; thickness $\mathrm{Si}_{3} \mathrm{~N}_{4}$ film $=250 \mathrm{~nm}$ ). The vertical dotted lines indicate the borders of the $\mathrm{Si}_{3} \mathrm{~N}_{4}$ mask. (b) XTEM image of LOPOS 3. (c) XTEM image of LOPOS 2.

stress in the substrate. The thickness of the $\mathrm{Si}_{3} \mathrm{~N}_{4}$ film also affects the magnitude of the horizontal force $f$. Indeed, $f$ is the film-edge-induced force and can be expressed as $f$ $=\sigma_{f} h$ where $\sigma_{f}$ is the tensile stress in the film and $h$ the film thickness. ${ }^{11,13}$ As discussed above, for broad lines the stress in the center $\Delta \omega_{\text {center }}$, is linearly related to $\sigma_{x x}$ and equal to $\Delta \omega_{3}$ [Eq. (18)]. Because $\sigma_{x x}$ is proportional to $f$ 
[Eq. (8)], $\Delta \omega_{\text {center }}$ can directly be related to $f:$ Large $\Delta \omega_{\text {center }}$ corresponds with large $f$ and small shifts with small $f$. To test these predictions, micro-Raman measurements were performed on two LOPOS structures with different $\mathrm{Si}_{3} \mathrm{~N}_{4}$ film thicknesses: LOPOS 2 and LOPOS 3 (see Table I). All the other process parameters were the same for both samples. Figure 9(a) shows the Raman shift obtained for a scan across a $5-\mu \mathrm{m}$ line of the LOPOS 2 sample and the LOPOS 3 sample, with laser light wavelength of $457.9 \mathrm{~nm}$. The experiment clearly shows that $\Delta \omega_{\min }$ is smaller in the LOPOS 2 sample (small $h$ ) than in the LOPOS 3 sample (large $h$ ). Also $\Delta \omega_{\text {center }}$ was found to decrease with decreasing $\mathrm{Si}_{3} \mathrm{~N}_{4}$ film thickness. From five measurements the following mean values were obtained: LOPOS $2(h=156 \mathrm{~nm}): \Delta \omega_{\min }=0.86 \pm 0.09 \mathrm{~cm}^{-1}$ and $\Delta \omega_{\text {center }}=0.13 \pm 0.04 \mathrm{~cm}^{-1}$; LOPOS $3(h=250 \mathrm{~nm})$ : $\Delta \omega_{\min }=1.35 \pm 0.07 \mathrm{~cm}^{-1}$ and $\Delta \omega_{\text {center }}=0.5 \pm 0.22$ $\mathrm{cm}^{-1}$. Assuming uniaxial stress, the following values for $\sigma_{x x}$ are found [Eq. (18)]: $\sigma_{x x}($ LOPOS 2) $\cong-0.07$ $\mathrm{GPa}<\sigma_{x x}$ (LOPOS 3$) \cong-0.26 \mathrm{GPa}$. These results confirm the model predictions very well and show that thicker $\mathrm{Si}_{3} \mathrm{~N}_{4}$ films result in larger $\Delta \omega_{\min }$ and $\Delta \omega_{\text {center. }}$. Figures $9(\mathrm{~b})$ and Fig. 9 (c) show XTEM pictures of LOPOS 2 and LOPOS 3, respectively. The difference in thickness of the $\mathrm{Si}_{3} \mathrm{~N}_{4}$ film is clearly shown and also the effect of this thickness on bending. The bending of the thicker film is considerably smaller than the bending of the thinner film. As a result, the length of the BB $\left(L_{\mathrm{bb}}\right)$ is influenced: Thinner films give longer, smoother BBs. XTEM observations showed that $L_{\mathrm{bb}}($ LOPOS 2$)=480 \mathrm{~nm}$ and $L_{\mathrm{bb}}($ LOPOS 3$)=340 \mathrm{~nm}$. These observations suggest that the stress at the $\mathrm{BB}$ is strongly related to the shape and length of the BB: Long, smooth BBs correspond with small stress and thus small $\Delta \omega_{\min }$, whereas short steep BBs give rise to high stress and thus high $\Delta \omega_{\text {min }}$.

\section{E. LOPOS-LOCOS}

To verify the relation between BBs length and stress, micro-Raman experiments were performed on a conventional LOCOS (LOCOS 1) and a LOPOS (LOPOS 1) structure and the results were compared with XTEM observations [Figs. 10(a)-10(c)]. The width of the scanned lines is $5 \mu \mathrm{m}$. The measurements were performed with an excitation wavelength of $457.9 \mathrm{~nm} . \Delta \omega_{\min }$ is found to be larger in the LOPOS than in the LOCOS structures, indicating higher stresses in the vicinity of the LOPOS BBs end. The XTEM image show that the BB in the LOCOS sample is longer and smoother than the BB in the LOPOS sample, which confirms that $\Delta \omega_{\min }$ is correlated with BBs length and shape. $\Delta \omega_{\text {center }}$ is found to be larger in the LOCOS sample than in the LOPOS sample. Assuming uniaxial stress in the silicon substrate at this position, the following stress values are obtained [Eq. (18)]: $\sigma_{x x}($ LOPOS $) \cong-0.34 \mathrm{GPa}<\sigma_{x x}($ LOCOS $) \cong$ $-0.52 \mathrm{GPa}$. The only difference between both samples is the presence of a poly-Si layer $(50 \mathrm{~nm})$ in the LOPOS structure. Poly-Si and single $c$-Si have about the same thermal-expansion coefficient. However, poly-Si exhibits an in-
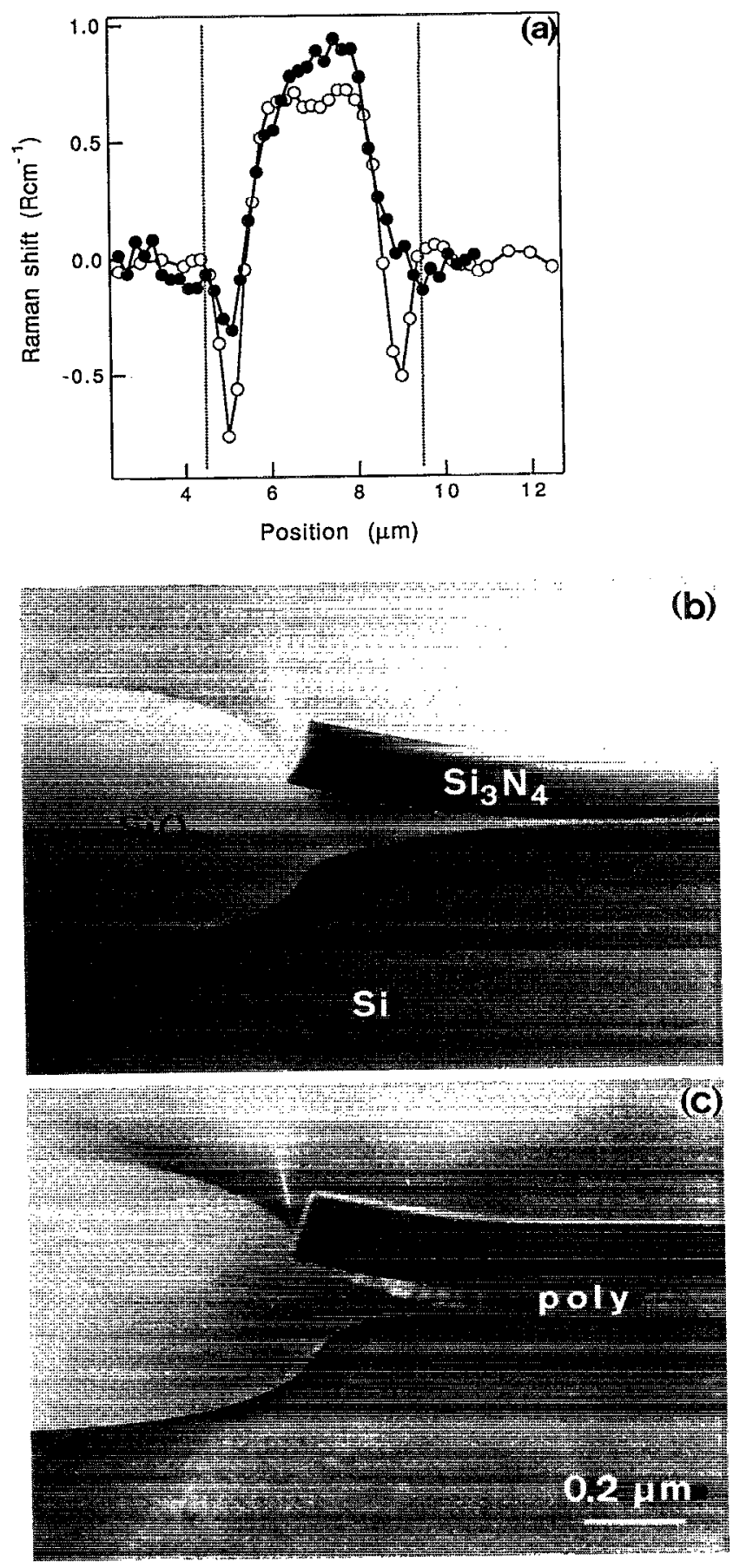

FIG. 10. (a) Raman frequency shift obtained during a scan across a 5- $\mu \mathrm{m}$ line of the LOPOS 1 sample (-O-) and the LOCOS 1 sample (- - ). The vertical dotted lines indicate the borders of the $\mathrm{Si}_{3} \mathrm{~N}_{4}$ mask. (b) XTEM image of LOCOS 1; (c) XTEM image of LOPOS 1.

trinsic compressive stress of about $-0.2 \mathrm{GPa}^{17}$ This stress partly compensates for the tensile stress in the $\mathrm{Si}_{3} \mathrm{~N}_{4}$ layer, resulting in a smaller compressive stress in the underlaying silicon substrate compared to the LOCOS sample.

\section{CONCLUSIONS}

The present results show that micro-Raman spectroscopy gives detailed information about local stress distribu- 
tion in LOCOS and LOPOS isolation structures. It is shown that an increase of the thickness of the $\mathrm{Si}_{3} \mathrm{~N}_{4}$ film and the corresponding reduction of the length of the $\mathrm{BB}$ result in an important increase of the local stress at the BBs edge. LOCOS structures with longer and smoother BBs clearly show smaller stress at this position. The stress in the Si substrate under the center of the oxidation mask lines is found to be compressive and to increase with decreasing linewidth. However, the influence of the large tensile stress at the BBs edge on the stress at the center of the lines also increases so that for smaller lines a decrease of the Raman shift and correlated stress is observed. By using different laser wavelengths information of the variation of stress with depth is obtained, indicating a decrease of the stress in depth, with a larger gradient at the BBs edge than at the center of the lines. Despite the fact that a complete fit of the simple planar stress model to the Raman data is not possible, important information can be extracted. Further progress can be expected by refinement of the model, e.g., by assuming a vertical force distribution instead of one vertical force.

\section{ACKNOWLEDGMENTS}

The authors acknowledge the Belgian Fund for Scientific Research (NFWO), the Spanish Ministry of Science and Education, and the Swedish Board of Technical Development. The Raman spectroscopy measurements were performed on the equipment of the K. U. Leuven (MTM department) and the XTEM work was performed on the equipment of the University of Antwerp (UCA).
${ }^{1}$ K. Kobayashi, Y. Inoue, T. Nishimura, M. Hirayana, Y. Akasaka, T. Kato, and S. Ibuki, J. Electrochem. Soc. 137, 1987 (1990).

${ }^{2} Y$. Inoue, T. Nishimura, and Y. Akasaka, in Advanced Materials for $U L S I$, edited by M. Scott, Y. Akasaka, and R. Reif (The Electrochemical Society, Pennington, NJ, 1988), p. 233.-

${ }^{3} \mathrm{~K}$. Brunner, G. Abstreiter, B. O. Kolbesen, and H. W. Meul, in 1989 Proceedings INFOS, edited by F. Koch and A. Spitzen, 1989, p. 116.

${ }^{4}$ S. Nadahara, S. Kambayashi, M. Watanabe, and T. Nakakubo, in Extended Abstracts of the 19th Conference on Solid State Devices and Materials, Tokyo, 1987, p. 327.

${ }^{5}$ S. Kambayashi, T. Hamasaki, T. Nakakubo, W. Watanabe, and H. Tango, in Extended Abstracts of the 19th Conference on Solid State Devices and Materials, Tokyo, 1986, p. 415.

${ }^{6}$ R. Loudon, Adv. Phys. 13, 423 (1964).

${ }^{7}$ S. Ganesan, A. A. Maradudin, and J. Oitınaa, J. Ann. Phys. 56, 556 (1970).

${ }^{8}$ E. Anastassakis, and E. Burstein. J. Phys. Chem. Solids 32, 563 (1971).

${ }^{9}$ E. Anastassakis, in Proceedings ISPPME' 85 , Farna, Bulgaria: Physical Problems in Microelectronics, edited by J. Kassabov (World Scientific Publishing Co., Singapore, 1985), p. 128.

${ }^{10} \mathrm{~S}$. P. Timoshenko and J. N. Goodier, in Theory of Elasticity, 2nd edition, edited by R. H. Phelps (McGraw-Hill, New York, 1951).

${ }^{11}$ R. Zeyfang, Solid-State Electron. 14, 1035 (1971).

${ }^{12}$ S. M. Hu, J. Appl. Phys. 50, 4661 (1979).

${ }^{13}$ J. Vanhellemont, S. Amelinckx, and C. Claeys, J. Appl. Phys. 61, 2170 (1987).

${ }^{14}$ E. Anastassakis, in Proceedings Vol. 90-11: Analytical Techniques for Semiconductor Materials and Process Characterization, edited by B. O. Kolbesen, D. V. Mc Caughian, and W. Vandervorst (The Electrochenical Society, Pennington, NJ, 1990), p. 298.

${ }^{15} \mathrm{~J}$. Vanhellemont, C. Claeys, J. Van Landuyt, and S. Amelinckx, in 1985 Proceedings GADEST, edited by $\mathrm{H}$. Richter, p. 255.

${ }^{16}$ I. De Wolf, J. Vanhellemont, A. Romano-Rodríguez, H. Norström, and H. E. Maes, in Proceedings of the Third International Symposium on ULSI Science and Technology, The Electrochemical Society, 179th meeting, Washington, DC, May 5-10, 1991.

${ }^{17} \mathrm{~S} . \mathrm{M} . \mathrm{Hu}$, in Proceedings of the 2nd International Symposium on Process Physics and Modeling in Semiconductor Technology (The Electrochemical Society, Pennington, NJ, 1991), p. 548. 